\title{
BIBECHANA
}

A Multidisciplinary Journal of Science, Technology and Mathematics ISSN 2091-0762 (Print), 2382-5340 (0nline)

Journal homepage: http://nepjol.info/index.php/BIBECHANA

Publisher: Research Council of Science and Technology, Biratnagar, Nepal

\section{Analytical exact solution of telegraph equation using HPM}

\section{Jamshad Ahmad $^{* 1}$ and Ghulam Mohiuddin ${ }^{2}$}

${ }^{1}$ Department of Mathematics, Faculty of Sciences, University of Gujrat, Pakistan

${ }^{2}$ Department of Mathematics, NCBA \& E, Pakistan

*Email: jamshadahmadm@gmail.com

Article history: Received 1 March, 2016; Accepted 4 August, 2016

DOI: http://dx.doi.org/10.3126/bibechana.v14i0.15411

This work is licensed under the Creative Commons CC BY-NC License.

https://creativecommons.org/licenses/by-nc/4.0/

(c) (i) (8)

Abstract

In this paper, exact solutions of different variants of second order hyperbolic telegraph equation are investigated with Homotopy Perturbation Method (HPM). The results determined by the proposed method are quite satisfactory and shows that HPM technique is very effective and useful for solving the nonlinear partial differential equations (PDEs) with given initial or boundary conditions. The proposed iterative scheme finds the solution without any discretization, linearization or restrictive assumptions.

Keywords: Telegraph equation; Homotopy perturbation method.

\section{Introduction}

Hyperbolic PDEs are the area of interest of many mathematicians because of their applications in many applied sciences for example wave equation and telegraph equation. The telegraph equation was first introduced by kirchoff in 1857. But it was first examined by Poincare in 1893. The telegraph equation has both wave motion and diffusion properties. Telegraph equation describes various phenomena in many appliedfields, for example in fluid dynamics it shows the random motion of the particle, travelling of electromagnetic waves in superconducting media and propagation of pressure waves occurring in pulsatile blood flow in arteries [1].

In the last few years, many techniques have been introduced for the exact and numerical solutions of the nonlinear PDEs, such as the ADM [2], Exponential function method [3,4], Homotopy Analysis Method (HAM)[5-7] and Variational Iteration Method (VIM)[8-10]. 
In this work, we use homotopy perturbation method (HPM) for solving hyperbolic telegraph equation. Besides from the other techniques, the nonlinear PDEs are solved easily and efficiently without transforming or linearizing the equation by using the Homotopy Perturbation Method (HPM) [10]. It gives the solution with great accuracy, less calculations and avoiding of unnecessary assumptions. The HPM was first proposed by He and it is applied to solve a wide class of nonlinear PDEs and ODEs effectively, more easily and much accurately with approximations converging rapidly to accurate solutions.

\section{Basic Idea of Homotopy Perturbation Method (HPM)}

Consider the following non-linear differential equation

$$
A(u)-f(r)=0 \quad r \in \Omega,
$$

with the boundary conditions of

$$
B\left(u, \frac{\partial u}{\partial n}\right)=0 \quad r \in \Gamma,
$$

where $A, B, f(r)$ and $\Gamma$ are differential operator, boundary operator, known analytic function and the boundary of the domain $\Omega$, respectively.

The operator $A(u)$ can be divided into a linear part $L(u)$ and a non-linear part $N(u)$.

Therefore Eq.(1) can be rewritten as

$$
L(u)+N(u)-f(r)=0 .
$$

In case the nonlinear Eq. (1) has no small parameter, we can construct the following homotopy,

$$
H(v, p)=L(v)-L\left(u_{0}\right)+p L\left(u_{0}\right)+p[N(v)-f(r)]=0 .
$$

Here $p$ is homotopy parameter.

According to the Homotopy Perturbation Method, the approximate solution of Eq. (4) can be expressed as a series of the power $p$ i.e.

$$
u=\lim _{p \rightarrow 1}\left(u_{o}+p u_{1}+p^{2} u_{2}+p^{3} u_{3}+\ldots\right) \text {. }
$$

When Eq. (4) corresponds to Eq. (1), (5) becomes the approximate solution of Eq. (1).

\section{Numerical Examples}

In this section we give some numerical examples to check the efficiency and accuracy of the proposed method by solving some hyperbolic telegraph equation.

Example 3.1 Consider the following Hyperbolic Telegraph equation

$$
u_{t t}+u_{t}+u=u_{x x} \text {, }
$$

with the initial conditions

$$
u(x, 0)=e^{x}, u_{t}(x, 0)=-e^{x} .
$$

According to the above defined procedure, we have

$$
u(x, t)=e^{x}-t e^{x}+L^{-1}\left[u_{x x}-u-u_{t}\right]
$$




$$
u_{0}+p u_{1}+p^{2} u_{2}+p^{3} u_{3}+\ldots=e^{x}-t e^{x}+p L^{-1}\left[\begin{array}{l}
\left(u_{0}+p u_{1}+p^{2} u_{2}+p^{3} u_{3}+\ldots\right)_{x x}- \\
\left(u_{0}+p u_{1}+p^{2} u_{2}+p^{3} u_{3}+\ldots\right)- \\
\left(u_{0}+p u_{1}+p^{2} u_{2}+p^{3} u_{3}+\ldots\right)_{t}
\end{array}\right]
$$

Equating powers of $p$

$$
\begin{array}{ll}
p^{0}, & u_{0}(x, t)=e^{x}-t e^{x} \\
p^{1}, & u_{1}(x, t)=L^{-1}\left[\left(u_{0}\right)_{x x}-u_{0}-\left(u_{0}\right)_{t}\right] \\
u_{1}(x, t)=\frac{t^{2}}{2 !} e^{x} \\
p^{2}, & u_{2}(x, t)=L^{-1}\left[\left(u_{1}\right)_{x x}-u_{1}-\left(u_{1}\right)_{t}\right] \\
& u_{2}(x, t)=-\frac{t^{3}}{3 !} e^{x} \\
p^{3}, & u_{3}(x, t)=L^{-1}\left[\left(u_{2}\right)_{x x}-u_{2}-\left(u_{2}\right)_{t}\right] \\
\vdots & u_{3}(x, t)=\frac{t^{4}}{4 !} e^{x} \\
\end{array}
$$

and so on. The closed form solution is

$$
\begin{aligned}
& u(x, t)=e^{x}-t e^{x}+\frac{t^{2}}{2 !} e^{x}-\frac{t^{3}}{3 !} e^{x}+\frac{t^{4}}{4 !} e^{x}+\ldots \\
& u(x, t)=e^{x-t} .
\end{aligned}
$$

This is the exact solution of the Eq. (6) as given by [11].

Example 3.2 Consider the following Telegraph Eq.

$$
\begin{aligned}
& u_{t t}+\alpha u_{t}+\beta u=u_{x x} \\
& \alpha=4, \quad \beta=2,
\end{aligned}
$$

with the initial conditions

$$
u(x, 0)=\sin x, u_{t}(x, 0)=-\sin x .
$$

According to the above defined procedure, we have

$$
\begin{gathered}
u(x, t)=\sin x-t \sin x+L^{-1}\left[u_{x x}-2 u-4 u_{t}\right] \\
u_{0}+p u_{1}+p^{2} u_{2}+p^{3} u_{3}+\ldots=\sin x-t \sin x+p L^{-1}\left[\begin{array}{c}
\left(u_{0}+p u_{1}+p^{2} u_{2}+p^{3} u_{3}+\ldots\right)_{x x} \\
-2\left(u_{0}+p u_{1}+p^{2} u_{2}+p^{3} u_{3}+\ldots\right) \\
-4\left(u_{0}+p u_{1}+p^{2} u_{2}+p^{3} u_{3}+\ldots\right)_{t}
\end{array}\right]
\end{gathered}
$$

Consequently,

$$
p^{0}, \quad u_{0}(x, t)=\sin x-t \sin x
$$




$$
\begin{aligned}
& p^{1}, \quad u_{1}(x, t)=L^{-1}\left[\left(u_{0}\right)_{x x}-2 u_{0}-4\left(u_{0}\right)_{t}\right] \\
& u_{1}(x, t)=\sin x\left[\frac{t^{2}}{2 !}+\frac{3 t^{3}}{3 !}\right] \\
& p^{2}, \quad u_{2}(x, t)=L^{-1}\left[\left(u_{1}\right)_{x x}-2 u_{1}-4\left(u_{1}\right)_{t}\right] \\
& u_{2}(x, t)=-\sin x\left[\frac{4 t^{3}}{3 !}+\frac{15 t^{4}}{4 !}+\frac{9 t^{5}}{5 !}\right]
\end{aligned}
$$

And so on. The closed form solution is

$$
\begin{aligned}
& u(x, t)=\sin x-t \sin x+\sin x\left[\frac{t^{2}}{2 !}+\frac{3 t^{3}}{3 !}\right]-\sin x\left[\frac{4 t^{3}}{3 !}+\frac{15 t^{4}}{4 !}+\frac{9 t^{5}}{5 !}\right]+\ldots \\
& u(x, t)=\sin x\left[1-t+\frac{t^{2}}{2 !}-\frac{t^{3}}{3 !}+\ldots\right] \\
& u(x, t)=\sin x \cdot e^{-t}
\end{aligned}
$$

This is exact solution [11].

Example 3.3 Consider the following Telegraph equations

$$
\begin{aligned}
& u_{t t}+\alpha u_{t}+\beta u=u_{x x}+f(x, t), \\
& \alpha=\beta=1 \quad, f(x, t)=x^{2}+t-1,
\end{aligned}
$$

with the initial conditions

$$
u(x, t)=x^{2}, u_{t}(x, t)=1 .
$$

According to the above defined procedure, we have

$$
\begin{aligned}
& u(x, t)=x^{2}\left(1+\frac{t^{2}}{2 !}\right)+t-\frac{t^{2}}{2 !}+\frac{t^{3}}{3 !}+L^{-1}\left[L\left(u_{x x}-u-u_{t}\right)\right] \\
& u_{0}+p u_{1}+p^{2} u_{2}+p^{3} u_{3}+\ldots=x^{2}\left(1+\frac{t^{2}}{2 !}\right)+t-\frac{t^{2}}{2 !}+\frac{t^{3}}{3 !}+p L^{-1}\left[\begin{array}{l}
\left(u_{0}+p u_{1}+p^{2} u_{2}+p^{3} u_{3}+\ldots\right) \\
-\left(u_{0}+p u_{1}+p^{2} u_{2}+p^{3} u_{3}+\ldots\right) \\
-\left(u_{0}+p u_{1}+p^{2} u_{2}+p^{3} u_{3}+\ldots\right)_{t}
\end{array}\right]
\end{aligned}
$$

Equating powers of $p$ 


$$
\begin{array}{ll}
p^{0}, & u_{0}(x, t)=x^{2}\left(1+\frac{t^{2}}{2 !}\right)+t-\frac{t^{2}}{2 !}+\frac{t^{3}}{3 !} \\
p^{1}, & u_{1}(x, t)=L^{-1}\left[\left(u_{0}\right)_{x x}-u_{0}-\left(u_{0}\right)_{t}\right] \\
u_{1}(x, t)=-x^{2}\left[\frac{t^{2}}{2 !}+\frac{t^{3}}{3 !}+\frac{t^{4}}{4 !}\right]+\frac{t^{2}}{2 !}+\frac{2 t^{4}}{4 !}-\frac{t^{5}}{5 !} \\
p^{2}, \quad u_{2}(x, t)=L^{-1}\left[\left(u_{1}\right)_{x x}-u_{1}-\left(u_{1}\right)_{t}\right] \\
u_{2}(x, t)=x^{2}\left[\frac{t^{3}}{3 !}+\frac{2 t^{4}}{4 !}+\frac{2 t^{5}}{5 !}+\frac{t^{6}}{6 !}\right]-\frac{t^{3}}{3 !}-\frac{3 t^{4}}{4 !}-\frac{4 t^{5}}{5 !}-\frac{3 t^{6}}{6 !}+\frac{t^{7}}{7 !} \\
p^{3}, \quad u_{3}(x, t)=L^{-1}\left[\left(u_{2}\right)_{x x}-u_{2}-\left(u_{2}\right)_{t}\right] \\
u_{3}(x, t)=-x^{2}\left[\frac{t^{4}}{4 !}+\frac{3 t^{5}}{5 !}+\frac{4 t^{6}}{6 !}+\frac{3 t^{7}}{7 !}+\frac{t^{8}}{8 !}\right]+\frac{t^{4}}{4 !}+\frac{4 t^{5}}{5 !}+\frac{5 t^{6}}{6 !}+\frac{3 t^{7}}{7 !}-\frac{t^{9}}{9 !}
\end{array}
$$

And so on. The series solution is

$$
\begin{aligned}
& u(x, t)=x^{2}\left(1+\frac{t^{2}}{2 !}\right)-x^{2}\left[\frac{t^{2}}{2 !}+\frac{t^{3}}{3 !}+\frac{t^{4}}{4 !}\right]+x^{2}\left[\frac{t^{3}}{3 !}+\frac{2 t^{4}}{4 !}+\frac{2 t^{5}}{5 !}+\frac{t^{6}}{6 !}\right]-x^{2} \\
& {\left[\frac{t^{4}}{4 !}+\frac{3 t^{5}}{5 !}+\frac{4 t^{6}}{6 !}+\frac{3 t^{7}}{7 !}+\frac{t^{8}}{8 !}\right]+t-\frac{t^{2}}{2 !}+\frac{t^{3}}{3 !}+\frac{t^{2}}{2 !}+\frac{2 t^{4}}{4 !}-\frac{t^{5}}{5 !}-\frac{t^{3}}{3 !}-\frac{3 t^{4}}{4 !}-\frac{4 t^{5}}{5 !}-\frac{3 t^{6}}{6 !}+\frac{t^{7}}{7 !}+\ldots}
\end{aligned}
$$

The closed form solution is

$$
u(x, t)=x^{2}+t
$$

This is the exact solution as given by [11].

Example 3.4 Consider the following Telegraph equation

$$
u_{t t}=u_{x x}-u-2 u_{t}
$$

with the initial conditions

$$
u(x, 0)=\cosh (x)-1, u_{t}(x, 0)=1 .
$$

According to the above defined procedure, we have

$$
\begin{aligned}
& u(x, t)=\cosh (x)+1-t+L^{-1}\left[u_{x x}-u-2 u_{t}\right], \\
& u_{0}+p u_{1}+p^{2} u_{2}+p^{3} u_{3}+\ldots=\cosh (x)+1-t+p L^{-1}\left[\begin{array}{l}
\left(u_{0}+p u_{1}+p^{2} u_{2}+p^{3} u_{3}+\ldots\right)_{x x}- \\
\left(u_{0}+p u_{1}+p^{2} u_{2}+p^{3} u_{3}+\ldots\right)- \\
\left(u_{0}+p u_{1}+p^{2} u_{2}+p^{3} u_{3}+\ldots\right)_{t}
\end{array}\right]
\end{aligned}
$$

Consequently, we have 


$$
\begin{array}{ll}
p^{0}, & u_{0}(x, t)=\cosh (x)+1-t \\
p^{1}, & u_{1}(x, t)=L^{-1}\left[\left(u_{0}\right)_{x x}-u_{0}-2\left(u_{0}\right)_{t}\right] \\
& u_{1}(x, t)=-\frac{t^{2}}{2 !}-\frac{t^{3}}{3 !} \\
p^{2}, & u_{2}(x, t)=L^{-1}\left[\left(u_{1}\right)_{x x}-u_{1}-2\left(u_{1}\right)_{t}\right] \\
& u_{2}(x, t)=\frac{2 t^{3}}{3 !}+\frac{3 t^{4}}{4 !}+\frac{t^{5}}{5 !} \\
p^{3}, & u_{3}(x, t)=L^{-1}\left[\left(u_{2}\right)_{x x}-u_{2}-2\left(u_{2}\right)_{t}\right] \\
\vdots, & u_{3}(x, t)=-\frac{4 t^{4}}{4 !}-\frac{8 t^{5}}{5 !}-\frac{5 t^{6}}{6 !}-\frac{t^{7}}{7 !} \\
&
\end{array}
$$

and so on. The series solution is

$$
\begin{aligned}
& u(x, t)=\cosh (x)-\left[1-t+\frac{t^{2}}{2 !}-\frac{t^{3}}{3 !}+\frac{t^{4}}{4 !}+\ldots\right] \\
& u(x, t)=\cosh (x)-e^{-t} .
\end{aligned}
$$

This is exact solution as was found in [12].

\section{Conclusion}

We have applied HPM for calculating the exact solution of second order hyperbolic telegraph equation. The results obtained by this method are quite satisfactory and are in accordance with the other techniques. The results gained through HPM prove that this is a powerful method for finding the exact.

\section{References}

[1] S. Y. Lai, J. Appl. Math.. Mech. 43(7) (1997) 657-662

[2] G. Adomian, Solving Frontier Problems of Physics, the Decomposition Method, Kluwer Academic Publishers, Dordrecht, (1994) pp. 372.

http://dx.doi.org/10.1007/978-94-015-8289-6

[3] J. He and M. Abdou, New periodic solutions for nonlinear evolution equations using Exp -function method, Chaos, Solutions and Fractals, 34 (2007) 1421-1429

http://dx.doi.org/10.1016/j.chaos.2006.05.072

[4] Q. M. Hassan S. T. Mohyud-Din, Exp-function method using modified Riemann-Liouville derivative for Burger's equations of fractional-order. QScience Connect: (2013).

[5] S. J. Liao, Notes on Homotopy analysis method, Some definitions and theorems, Common Nonlinear science and Numerical Simulations, 14 (2009) 983-997.

[6] A. Molabahrami, F. Khani, Real world Applications 10 (2009) 589-600.

http://dx.doi.org/10.1016/j.nonrwa.2007.10.014 
Jamshad Ahamad and Ghulam Mohiuddin/ BIBECHANA 14 (2017) 30-36 : RCOST p.36 (Online Publication: Dec., 2016)

[7] A. Mastroberardino, Common Nonlinear Science and numerical simulations 16 (2011) 2730-2736. http://dx.doi.org/10.1016/j.cnsns.2010.10.004

[8] J. He, Applied Mathematics and Computations,114 (2000) 115-123. http://dx.doi.org/10.1016/S0096-3003(99)00104-6

[9] L. Xu, (Guest Editors) J. H. He and A.M. Wazwaz, Journal of Computational and Applied Mathematics,207 (2007) 1-2.

http://dx.doi.org/10.1016/j.cam.2006.07.021

[10] M. Dehghani et.al, International Journal of Numerical methods and Biomedical Engineering, 27 (2011) 219-231.

http://dx.doi.org/10.1002/cnm.1293

[11] J. He, International Journal of Non-Linear Science and Numerical Simulation, 135 (2003) 73-79.

[12] M. Dehghan, A. Shokri, A Numerical Method for Solving the Hyperbolic Telegraph Equation, Wiley Inter Science, (2007).

[13] M. Rawashdeh, N. A. Obeidat, An International Journal of Applied Mathematics \& Information Sciences, 8( 5) (2014) 2171-2176. 